\title{
ADQUISICIÓN DE DATOS DE UNA MATRIZ DE SENSORES DE GASES (E-NOSE), MEDIANTE MÓDULOS DE COMUNICACIÓN XBEE
}

\section{DATA ACQUISITION FROM AN ARRAY OF GAS SENSORS (E-NOSE), THROUGH XBEE COMMUNICATIONS MODULES}

\author{
MSc. Pablo Andrés Goméz Monsalve, PhD. Cristhian Manuel Durán Acevedo. \\ * Universidad de Pamplona, Facultad de Ingenierías y Arquitectura, Grupo de \\ Investigación en Sistemas Multisensoriales y Reconocimiento de Patrones. \\ Km1. Vía Bucaramanga, Pamplona, Norte de Santander, Colombia. \\ Tel.:57-7-5685303, Fax: (+577) 5685303 Ext. 164. \\ E-mail: \{pablo.gomez, cmduran \} @unipamplona.edu.co.
}

\begin{abstract}
Resumen: El presente artículo consiste en la implementación de un sistema de comunicación inalámbrica compuesto por los módulos XBee de la Serie 2, para la adquisición de señales en sistemas multisensoriales. Los módulos fueron acoplados a una cámara de medida compuesta básicamente de una matriz de 6 sensores de óxidos metálicos, con el objetivo de ser utilizados para el desarrollo de una nariz electrónica. Una interfaz gráfica fue realizada en Labview para establecer la comunicación entre el módulo inalámbrico y el sistema sensorial, con el fin de obtener la información relevante del conjunto de datos. Las señales de los sensores fueron almacenadas y visualizadas en tiempo real en un computador, para verificar el comportamiento del sistema.
\end{abstract}

Palabras clave: Labview, nariz electrónica, adquisición de datos, sensores de gases, XBee.

\begin{abstract}
This article consist in the implementation of a wireless communication system composed of XBee Series 2 modules for signal acquisition in multisensor systems. The modules were coupled to measurement chamber that consisting essentially of a matrix of metal oxide sensors in order to be used for development of an electronic nose. A graphical user interface was made LabView for communication between the wireless module and the sensory system, in order to obtain the relevant information of data set. The sensor signals were stored and displayed in real time on a computer, to verify the behavior of the system.
\end{abstract}

Keywords: Labview, electronic nose, data acquisition, gas sensors, XBee.

\section{INTRODUCCIÓN}

Hoy en día las comunicaciones inalámbricas en diferentes aplicaciones industriales tienen grandes ventajas a comparación de un sistema de cableado convencional, donde cada vez más proporcionan una mayor eficiencia y seguridad en cada uno de los procesos. Una de las principales ventajas que proporcionan estos tipos de sistemas, es el bajo coste de instalación y mantenimiento (Chen, 2012).
Las instalaciones industriales suelen ser por lo general rigurosas y costosas, con requisitos muy exigentes en cuanto al tipo y calidad del cableado. Por lo tanto, prescindir de los cables significa menor cantidad de elementos presentes en un sistema, sobre todo cuando se trata de modernizar o actualizar los componentes dentro de la industria (Christin, 2010). Por tal motivo un sistema de comunicación inalámbrica puede ser una solución para ser implementada en entornos donde se 
requieran instalar grandes líneas de cableado, ya sea sea por motivos de la ubicación de los componentes o por la misma geografía del lugar de trabajo (Lorwongtragool, 2013).

El presente estudio tiene como objetivo realizar la implementación de los módulos inalámbricos XBee, para establecer la comunicación entre los nodos de una matriz de sensores de gases comerciales, la cual hace parte del desarrollo de una nariz electrónica, con el fin de adquirir las señales de los sensores en tiempo real. Los resultados obtenidos fueron muy satisfactorios, puesto que da un gran abanico de posibilidades para ser implementado en diferentes procesos industriales (por ejemplo, sistema de detección de gases explosivos en minas de carbón, control del medio ambiente (es decir, medición de la calidad del aire), en sectores de la salud, etc).

\subsection{La Nariz Electrónica}

Se define como Nariz Electrónica (E-Nose), a un tipo de instrumento de medida que comprende de una matriz de sensores de gases químicos y un avanzado sistema de reconocimiento de patrones, capaz de reconocer aromas tanto simples como complejos (Durán, 2005).

Los sistemas de detección de gases tienen muchas limitaciones, debido a su diseño ya que sólo se basa en la interpretación de una señal predominante de un sensor, donde su sensibilidad está orientada a un tipo de gas o gases en particular, identificando sólo algunos tipos de gases o compuestos. Como un ejemplo de lo anterior, un sensor solo reacciona en la presencia del metano donde su acción es activar una alarma sonora la cual indica la presencia del gas. Hoy en día es posible detectar la concentración de dicho gas, pero no es posible estar al tanto si este gas proviene de una fuente natural o de una mezcla orgánica. Además, sólo se encuentra diseñado para detectar metano y no otros gases. Para esta situación, las narices electrónicas superan esta limitación, porque su procesamiento está basado en un proceso similar a la nariz humana, en donde su interpretación de las señales es un conjunto de datos, lo cual permite reconocer mezclas orgánicas. A su vez, puede aprender a discriminar y/o reconocer nuevos olores.

Estos sistemas identifican la muestra en conjunto, sin importarle los componentes individuales que conforman el olor de la muestra.
Mediante este concepto las narices electrónicas permiten aplicar técnicas de pre-procesamiento de datos y reconocimientos de patrones para la detección, identificación o cuantificación de un aroma (Durán, 2005; Bambang, 2012; Panigrahi, 2006).

\subsection{Protocolo de comunicación ZigBee}

La tecnología ZigBee básicamente es un protocolo de comunicaciones inalámbrico, basado en el estándar de comunicaciones para redes inalámbricas bajo el estándar IEEE_802.15.4 (Baronti, 2007). Es muy útil para desarrollar redes de sensores en entornos tales como: Industriales, médicos, minería, entre otros, donde las comunicaciones se realizan en el rango de los 2.4 GHz. La red ZigBee proporciona las siguientes características de comunicación: Un sólo coordinador, uno o más ruteadores y uno o varios dispositivos móviles finales. Este tipo de características puede ser configurado en un módulo XBee.

\subsection{Topologías de redes ZigBee}

Para el caso de las redes ZigBee no se realiza una distribución física de los componentes, si no una conexión lógica entre nodos de la red. Este tipo de conexiones lógicas son:

- $\quad$ Par (Pair) o Punto Punto.

- Estrella (Star).

- Malla (Mesh).

- Árbol de racimos (Cluster Tree).

La Figura 1 ilustra la distribución de cada una de las topologías lógicas de las redes ZigBee.

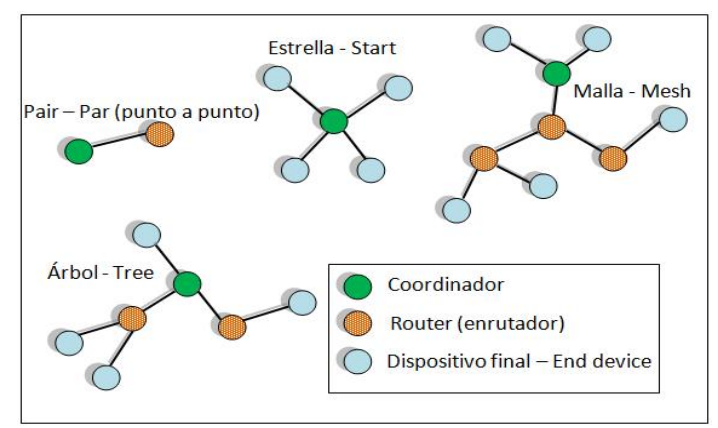

Fig. 1. Topologías lógicas redes ZigBee. (Plataformas Zigbee, 2014).

\subsection{Funciones del protocolo ZigBee}

ZigBee permite realizar tres funciones en una red de datos, las cuales son: Ruteo, creación de redes Ad Hoc y auto-regeneración (Longkang, 2011). 


\subsection{Direccionamiento de un nodo de red ZigBee}

Cada nodo en una red ZigBee necesita una información que lo identifique de los demás nodos adyacentes a él, donde esta información consiste en un número de serie de 64 bits y una dirección de 16 bits.

\subsection{Dirección PAN (Personal Area Network)}

Tiene como característica una dirección de 16 bits, donde hay 65536 direcciones disponibles y cada una puede generar otras 65536 direcciones para nodos debajo de ella (es decir, nodos hijos).

\subsection{El Módulo XBee}

Xbee es una "marca" de transceptores los cuales soportan el protocolo ZigBee y otros protocolos más. El modulo presenta dos tipos: Los disponibles con ZigBee o Serie 2 y los que no poseen ZigBee o Serie 1. La Tabla 1 describe las diferencias entre cada una de las series.

Tabla 1. Diferencias entre serie 1 y serie 2. (Digi, 2014).

\begin{tabular}{|c|c|c|}
\hline Característica & Serie 1 & Serie 2 \\
\hline $\begin{array}{l}\text { Alcance típico } \\
\text { (interior/urbano) }\end{array}$ & 30 metros & 40 metros \\
\hline $\begin{array}{c}\text { Mejor rango } \\
\text { (línea de Vista) }\end{array}$ & 100 metros & 120 metros \\
\hline $\begin{array}{c}\text { Corriente del } \\
\text { Transmisor } \\
\text { /Receptor }\end{array}$ & $45 / 50 \mathrm{~mA}$ & $40 / 40 \mathrm{~mA}$ \\
\hline $\begin{array}{l}\text { Firmware } \\
\text { (typical) }\end{array}$ & $\begin{array}{c}802.15 .4 \\
\text { punto a } \\
\text { punto }\end{array}$ & $\begin{array}{l}\text { ZB ZigBee } \\
\text { Malla }\end{array}$ \\
\hline $\begin{array}{c}\text { Pines de } \\
\text { entrada/salida } \\
\text { digital }\end{array}$ & $\begin{array}{c}8 \text { (plus } 1 \\
\text { entrada } \\
\text { solamente) }\end{array}$ & 11 \\
\hline $\begin{array}{l}\text { Pines de entrada } \\
\text { análoga }\end{array}$ & 7 & 4 \\
\hline $\begin{array}{c}\text { Pines de salida } \\
\text { análoga (PWM) }\end{array}$ & 2 & No \\
\hline $\begin{array}{l}\text { Baja potencia, bajo } \\
\text { ancho de banda, } \\
\text { bajo costo, } \\
\text { direccionable, } \\
\text { estandarizado, } \\
\text { pequeño popular }\end{array}$ & $\mathrm{Si}$ & $\mathrm{Si}$ \\
\hline $\begin{array}{l}\text { Firmware } \\
\text { Disponible }\end{array}$ & $\begin{array}{c}802.15 .4 \\
\text { (IEEE } \\
\text { estándar), } \\
\text { DigiMesh } \\
\text { (propietario) }\end{array}$ & $\begin{array}{c}\text { ZB (ZigBee } \\
\text { 2007), } \\
\text { ZNet } \\
2.5 \\
\text { (obsoleto) }\end{array}$ \\
\hline
\end{tabular}

\subsection{Modos de funcionamiento XBee}

Según la forma de intercambio de datos, XBee posee tres modos de funcionamiento: Modo transparente, Modo de comandos AT y Modo API. Según su fuente de TX/RX, los módulos pueden transmitir datos provenientes desde entradas de tipo serial, analógica y digital. La serie XBeepro permite mayores distancias de transmisión en un rango de 1600 metros con línea de vista (Digi, 2014).

\section{MODELO DE COMUNICACIÓN (APLICACIÓN EN MINAS)}

La topología de comunicación para el sistema inalámbrico es de tipo árbol, la cual se puede utilizar en espacios confinados. Por esta razón, es posible implementar la distribución de los sensores de una nariz electrónica, dentro de un lugar con espacio reducido, como es el caso de una mina (Ver figura 2). A su vez, es posible obtener la mejor comunicación entre los nodos del mismo tipo, tal y como se muestra en la Figura 3.

El modelo se implementó con una configuración tipo API (Application Programming Interface), el cual permite mayor información del nodo y así conocer el estatus del mismo.

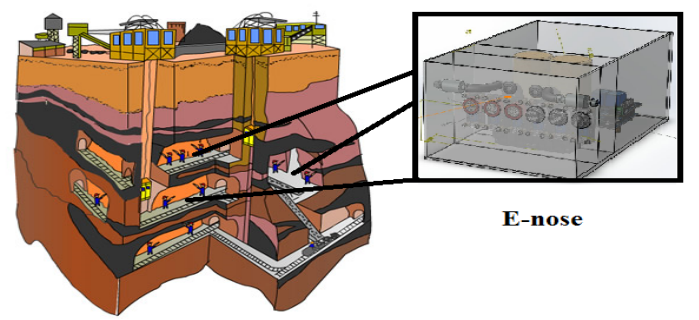

Fig. 2. Ubicación de los diferentes nodos dentro de una mina.

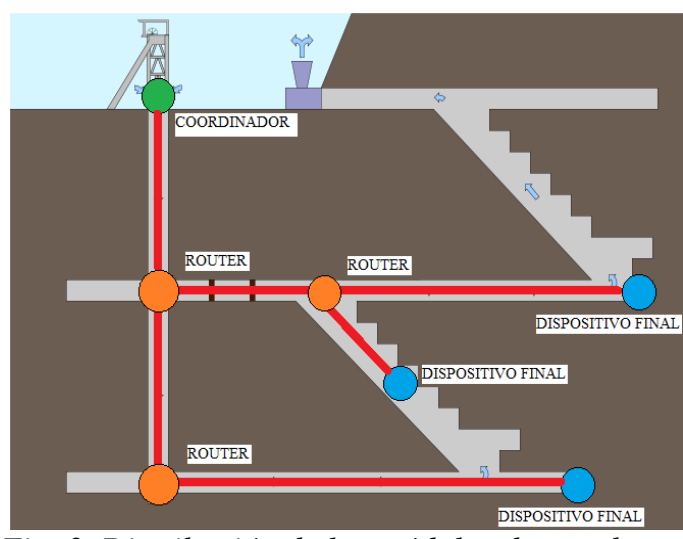

Fig. 3. Distribución de los módulos dentro de una mina. 
Debido a la complejidad de la geografía presentada dentro de una mina, la ubicación de los diferentes nodos varía en cuanto a la distribución de esta misma (por ejemplo: túneles, galerías, entre otros).

El modelo del proceso de la comunicación, consiste en generar una trama de datos de la característica para cada nodo (Ver figura 4).

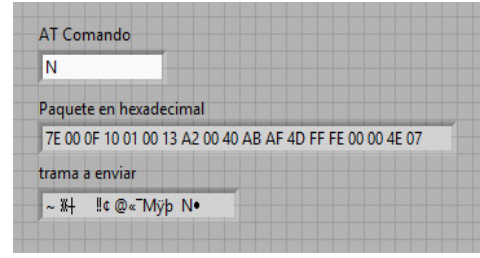

Fig. 4. Trama generada en Labview para enviar la letra $N$ a un nodo especifico.

Tal y como se observa en la figura 5, la trama es transmitida en modo serial al coordinador, el cual está interconectado a un adaptador Xbee-USB, este a su vez envía las peticiones al nodo preseleccionado y el nodo gestiona la petición dependiendo del caso (es decir, router o dispositivo final). Si se selecciona un dispositivo final lejano, se le indicará a un router predefinido el cual servirá como repetidor para que el coordinador reciba los datos del punto lejano.

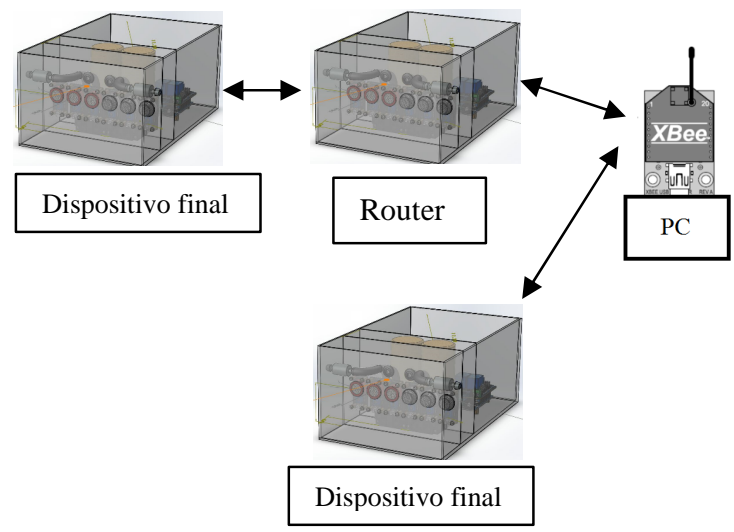

Fig. 5. Modelo de la comunicación entre E-nose.

\section{PRUEBAS DEL MODELO}

El prototipo de un nodo lo constituye principalmente de una matriz de 6 sensores de gases de tipo MQ de HwSensor, modelos: MQ3, MQ7, MQ6, MQ2, MQ8, MQ9; un módulo XBee, una Shield, un tarjeta de desarrollo Arduino (MEGA 2560), 2 bombas de purga y carga de la cámara de gases con los filtros de protección. En la figura 6 se observa la ubicación de los componentes.

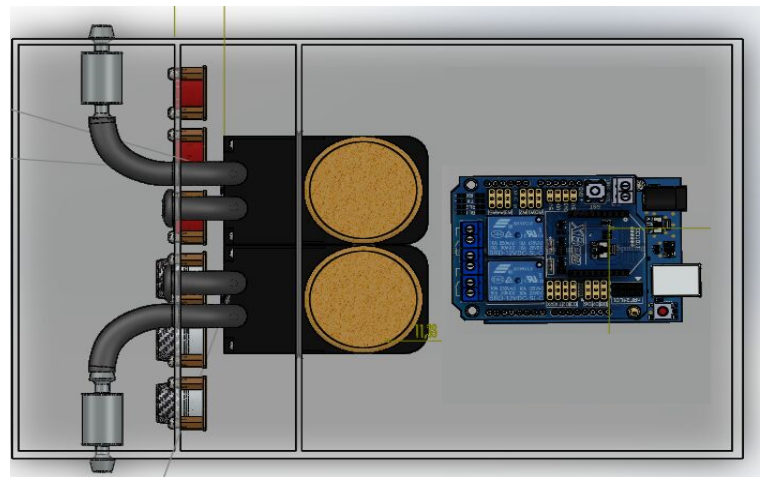

Fig. 6. Distribución de los componentes dentro de la caja que conforma uno de los nodos.

En la figura 7 se ilustra el circuito de acondicionamiento de la señal y la ecuación para el cálculo de la variación de la resistencia del sensor de gas (Rs), el cual fue usado en cada uno de los canales de la tarjeta de desarrollo. Donde:

$\mathrm{V}_{\mathrm{c}}=$ Voltaje de alimentación del sensor.

$\mathrm{V}_{\mathrm{h}}=$ Voltaje del heater .

$\mathrm{R}_{\mathrm{L}}=$ Resistencia de carga.

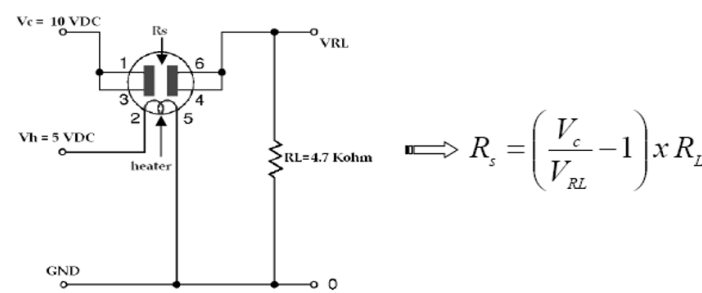

Fig 7. Circuito de acondicionamiento del Sensor. Fuente: (Figaro Engineering Inc, 2014).

A través del circuito de acondicionamiento de la señal fue posible acoplar cada una de las fuentes DC y sensores, necesarios para el buen funcionamiento de la nariz electrónica.

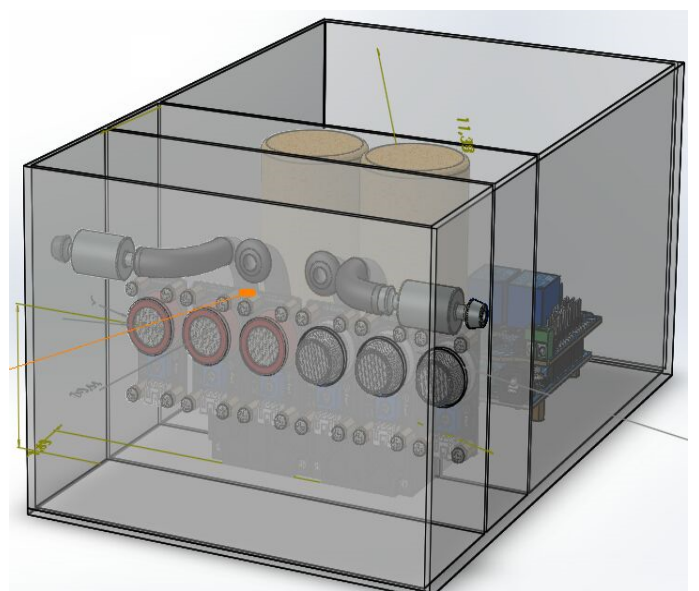

Fig 8. Distribución de los sensores dentro de la cámara. 
En la figura 8 se ilustra la distribución en forma lineal de los sensores en la cámara de medición, con el objetivo de permitir una mayor velocidad de reacción a los componentes de los gases en cada uno de los sensores. Una función muy importante es maximizar la eliminación de los mismos al momento de purgar la cámara.

\section{RESULTADOS}

En la Figura 9 se observa la interfaz gráfica de usuario, la cual permitió visualizar cada una de las variables correspondientes al modelo del prototipo de nariz electrónica, en el cual se generó una matriz de datos a partir de los cuatro sensores de gases (señales de color rojo, verde, azul y negro). Estas señales indican la no-selectividad en cuanto a la sensibilidad en cada uno de los sensores a los compuestos volátiles o gases. Las respuestas fueron adquiridas tomando como referencia el aire del ambiente y a una temperatura de 25 grados centígrados. Lo anterior es una de las principales características del buen funcionamiento de las narices electrónicas. Tal y como se observa en la figura, los sensores presentaron una buena sensibilidad y gran velocidad de respuesta al aire ambiente.

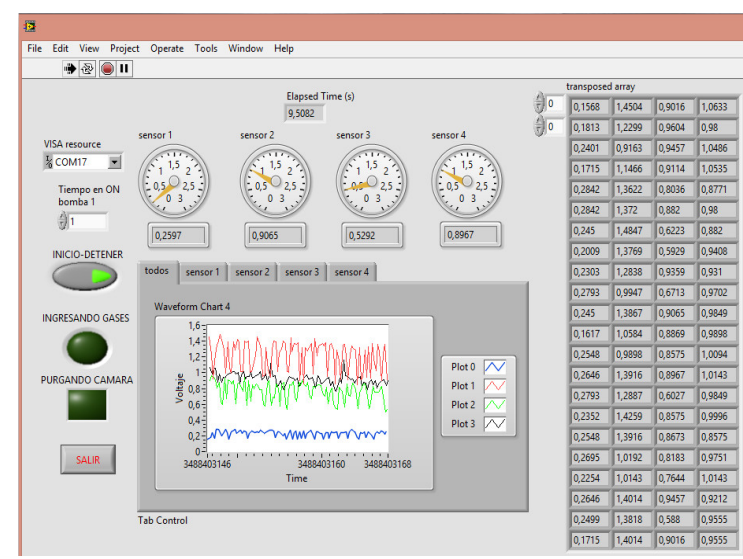

Fig. 9. Interfaz de adquisición de datos.

Las señales fueron adquiridas en valores de voltaje, dando como resultados una matriz de datos con información relevante y con poca afectación de ruido ni derivas en los sensores químicos.

\section{CONCLUSIONES}

Las pruebas realizadas con el sistema inalámbrico y la nariz electrónica demostraron la buena versatilidad de los componentes (Es decir, arduino, Módulos XBee, sensores químicos, etc).
Con este estudio se logró comprobar la confiabilidad de la aplicación, puesto que el modelo de comunicación inalámbrica, la programación y los medios utilizados sirvieron para almacenar y visualizar correctamente la información de las señales de los sensores.

La implementación del protocolo ZigBee provista por los XBee Series 2, permitió generar una red de datos confiable, facilitando la comunicación entre los nodos del sistema.

Mediante el funcionamiento del sistema inalámbrico en modo API, se pudo conocer los estados del nodo, las fallas del envió de información de las tramas y el módulo NI VISA, el cual se utilizó para la comunicación serial con LabView.

A partir de los resultados obtenidos con el sistema inalámbrico y los datos adquiridos de la nariz electrónica, es posible realizar aplicaciones en tiempo real para el monitoreo y control de diferentes procesos que requieran de un sistema multisensorial de tales características (por ejemplo, en el sector industrial, ambiental, agroindustrial, salud, etc).

Debido al reducido número de componentes utilizados en la realización de los nodos (es decir, mediante la cámara de gases), el tamaño de estos fue más reducido en comparación a otros sistemas encontrados comercialmente.

\section{REFERENCIAS}

Chen, S et al. (2012). "Analysis of the Power Consumption for Wireless Sensor Network Node Based on Zigbee", Procedia Engineering, Vol. 29, 2012, pp. 1994-1998.

Christin, D. et al. (2010). "Survey on Wireless Sensor Network Technologies for Industrial Automation: The Security and Quality of Service Perspectives", Magazine Future, Vol. 2, 2010, pp 96-125.

Lorwongtragool, P. et al. (2013). “A Zigbee-based wireless wearable electronic nose using flexible printed sensor array", Nanoelectronics Conference (INEC), IEEE 5th International, Singapore, 2013.

Durán, C. (2005). Diseño y optimización de los subsistemas de un sistema de olfato electrónico para aplicaciones agroalimentarias e industria, Tesis de Doctorado en Ingeniería Electrónica, Universidad Rovira i Virgili, Tarragona. 
Ampuero, S. and Bosset, J. "The electronic nose applied to dairy products: a review". Sensors and Actuators B: Chemical, Vol. 94, 2003, pp. $1-12$.

Bambang, C. et al. (2012). "Wireless e-Nose Sensor Node: State of the Art", Procedia Engineering, Vol. 41, 2012, pp. 1405-1411.

Panigrahi, S. (2006). "Design and development of a metal oxide based electronic nose for spoilage classification of beef', Sensors and Actuators B: Chemical. Vol. 119, November, 2006, pp. $2-$ 14.

Baronti, P. et al. (2007). "Wireless sensor networks: A survey on the state of the art and the 802.15.4 and zigBee standards", Computer Communications, Vol. 30, 2007, pp. 1655-1695

Longkang, W. et al. (2011). "Zigbee - Based Positioning System For Coal Miners", Procedia Engineering, Vol. 26, 2011, pp. 2406-2414.

\section{SITIOS WEB}

Digi, XBee ${ }^{\circledR} \mathrm{ZB}$ (2014). módulos RF zigbee pro http://www.digi.com/products/wirelesswired-embedded-solutions/zigbee-rfmodules/zigbee-mesh-module/xbee-zbmodule. (Consultado: 31/07/2014)

Figaro, Engineering Inc. (2014). Sensores de gases figaro. http://www.figarosensor.com/. (Consultado: 31/07/2014)

Plataformas Zigbee, (2014). Sistemas de control inalámbrico zigbee. Consultado: 20/08/2014.

http://plataformaszigbee.blogspot.com. 